\title{
Recomendaciones para la Categorización y Manejo de Pacientes con Traumatismos Faciales Durante Pandemia por COVID-19 (SARS-CoV-2). Revisión de la Literatura
}

\author{
Recommendations for the Categorization and Management of Patients with \\ Facial Trauma During a COVID-19 (SARS-CoV-2) Pandemic. Literature Review
}

\author{
Francisco Yévenes Souper'; Marlene Diaz Reiher'; Francisca Donoso Hofer ${ }^{2}$
}

\begin{abstract}
YÉVENES, S. F.; DIAZ, R. M. \& DONODO, H. F. Recomendaciones para la categorización y manejo de pacientes con traumatismos faciales durante pandemia por COVID-19 (SARS-CoV-2). Revisión de la literatura. Int. J. Odontostomat., 15(1):31-35, 2021.

RESUMEN: El Trauma facial como tal constituye un problema de relevancia en los centros hospitalarios de alta complejidad debido a las consecuencias funcionales y estéticas que pueden conllevar. Es por eso la necesidad de un diagnóstico, respuesta asertiva y manejo adecuado de éstos y más en los tiempos de una pandemia como el COVID-19. Se revisaron diferentes protocolos respecto a la categorización y manejo de esta patología tomando en cuenta la situación pandémica que nos afecta. El objetivo de este artículo es presentar y acercar estos protocolos, y así, lograr ser un aporte a los distintos profesionales dedicados a tratamientos del trauma facial a nivel hospitalario respecto a las medidas de seguridad tanto con todo el personal como con los pacientes
\end{abstract}

PALABRAS CLAVE: COVID-19, SARS-CoV-2, trauma facial, protocolo.

\section{INTRODUCCION}

Desde su inicio a finales del año 2019 en la ciudad de Wuhan (China), el SARS-CoV-2 (Severe acute respiratory síndrome coronavirus-2) más conocido actualmente como COVID-19, se ha presentado de una manera insidiosa y propagado rápidamente por los distintos continentes, siendo declarado como pandemia el día 11 de marzo de 2020 por la Organización Mundial de la Salud (2020).

La convivencia con el virus en todos los entornos clínicos y hospitalarios de salud presenta un reto a la adaptación, creación de protocolos y modelos asistenciales eficientes. Dentro de este mismo entorno, los procedimientos quirúrgicos se ven sumamente afectados, ya sea por la saturación de los servicios, la reorganización de los centros hospitalarios, o la reorganización de maquinas y personal para controlar la alta demanda consecuente al COVID-19, siendo obviamente cirugías electivas las primeras afectadas (Hsieh et al., 2020).
Otro punto importante en este ámbito es el porcentaje de contagio del personal de salud, sobre todo en especialidades que trabajan de cerca con el sistema respiratorio: Cirujanos Máxilofaciales, Cabeza y cuello, Otorrinos y broncopulmonares A la fecha de esta revisión, en España (uno de los países europeos más afectados) un $14 \%$ de los trabajadores de salud resultó positivo para COVID-19 mientras que en China, un reciente estudio reportó que un tercio de los trabajadores de salud sufrieron el contagio por COVID-19 (Federazione Nazionale degli Ordini dei Medici Chirurghi e degli Odontoiatri, 2020; Minder \& Peltier, 2020).

Son varias las incógnitas que se deben resolver aún sobre el desarrollo y alcance de esta pandemia en los servicios de salud y si bien, la resolución de las complicaciones respiratorias de los pacientes Covid-19 siguen siendo prioritarias, existen otras patologías y condiciones que deben comenzar

${ }^{1}$ Cirujano Dentista, Servicio de Cirugía Oral y Maxilofacial, Hospital San Juan de Dios, Santiago, Chile.

${ }^{2}$ Cirujano Maxilofacial, Servicio de Cirugía Oral y Maxilofacial, Hospital San Juan de Dios, Santiago, Chile. 
YÉVENES, S. F.; DIAZ, R. M. \& DONODO, H. F. Recomendaciones para la categorización y manejo de pacientes con traumatismos faciales durante pandemia por COVID-19 (SARS-CoV-2). Revisión de la literatura. Int. J. Odontostomat., 15(1):31-35, 2021.

a resolverse para no aumentar la morbi-mortalidad asociada.

Muchos casos de trauma máxilo facial no necesitan ser resueltos de manera urgente cuando no hay riesgo vital dado principalmente por el compromiso de la vía aérea, pero la postergación de la resolución quirúrgica afecta muchas veces la calidad de vida del paciente y su relación con el entono y con otras personas.

El objetivo de este trabajo es revisar la literatura publicada sobre la categorización y manejo del trauma facial, logrando un equilibrio entre la atención de un paciente en una situación determinada vs la protección del personal de salud mediante el uso juicioso de la información y recursos disponibles.

\section{PROTOCOLOS}

En los inicios de esta pandemia, varios grupos de cirujanos, de un amplio espectro de países recomendaron que los procedimientos electivos o no esenciales debían posponerse o cancelarse debido al COVID-19, debido a: utilización de recursos, riesgo de infección tanto para el personal como pacientes, disponibilidad de equipos quirúrgicos, etc. Si bien esta recomendación se sigue en muchos centros hospitalarios, ,no existe consenso con respecto a este tema, y por ejemplo The American College of Surgeons (ACS) emite una mirada más cautelosa al respecto publicando que la evaluación y categorización en este tipo de procedimientos debe ser valorada caso a caso.y que debe evaluar las medidas de protección frente a Covid19 y categorización de los pacientes con respecto a la infección viral y a la severidad del trauma (American College of Surgeons, 2020a).

\section{Medidas de protección frente a COVID-19}

En el contexto de una fractura del territorio máxilo facial, el manejo de las estructuras anatómicas presenta un riesgo particularmente alto por la posibilidad de presentar una carga viral alta y al aerosol formado en estructuras como la cavidad oral, nasal, mucosa orofaríngea entre otras.

En un estudio publicado por el Departamento de Otorrinolaringología en conjunto con los Cirujanos de Cabeza y Cuello de la Universidad de Minnesota optaron por tomar 2 conjuntos de precauciones en base a publicaciones por pate de la Universidad de Stanford (Hsieh et al.; Vukkadala et al., 2020).

- Precauciones extremas del aire: Se incluye un motor purificador de aire, ropa clínica resistente a fluidos y guantes quirúrgicos.

- Precauciones en mejoras del aire: Se incluye el uso de mascarillas N95, protector facial u ocular como antiparras, ropa clínica resistente a fluidos y guantes quirúrgicos (Hsieh et al.; Vukkadala et al.).

\section{Conocimiento status y categorización COVID-19 de los pacientes}

Dependiendo de cada país, sistema de salud y laboratorios, el resultado de la prueba de PCR para detectar partículas virales entre 24 y 48 horas (Kaplan et al., 2020). Si la gravedad de la fractura lo permite, lo ideal es contar con el resultado del examen del paciente antes de entrar a pabellón. La Universidad de Standford recomienda realizar dos pruebas para COVID-19 previo a la cirugía, distanciándolas por 24 horas de diferencia: si ambas pruebas resultan negativas se debe aplicar el protocolo de mejoras del aire; si ambas dan positivas, o sólo una se recomienda tomar las precauciones extremas.

La categorización y determinación del status COVID-19 del paciente debe ser utilizando todos los medios posibles, incluso sumando la radiografía torácica -preferiblemente TAC. Frente a la imposibilidad de determinar el estatus, tratarlo como un paciente positivo, lo cual es compartido por la Asociación española de cirujanos (Rubio-Pérez et al., 2020) y la American College of Surgeons, (2020b).

A nivel nacional se han publicado varias recomendaciones por parte del Colegio médico, Sociedad de cirujanos pediátricos y la Sociedad de Anestesiología chilena los cuales coinciden con los organismos internacionales: recomiendan evaluar al paciente lo más completamente posible preoperatoriamente, categorización quirúrgica y toma de exámenes PCR previo a cirugía (Sociedad Chilena de Cirugía Pediátrica, 2020).

\section{Evaluación y categorización de las fracturas}

Para evaluar y categorizar las fracturas deben evaluarse una serie de condiciones que puedan influir en la morbi-mortalidad del paciente en el corto, mediano y largo plazo. La decisión de operar de 
YÉVENES, S. F.; DIAZ, R. M. \& DONODO, H. F. Recomendaciones para la categorización y manejo de pacientes con traumatismos faciales durante pandemia por COVID-19 (SARS-CoV-2). Revisión de la literatura. Int. J. Odontostomat., 15(1):31-35, 2021.

manera urgente o de diferir la cirugía hasta contar con los exámenes necesarios e incluso esperar a que las condiciones del recinto de salud sean las óptimas no es fácil.

Para fracturas en que la morbilidad y/o mortalidad es significativa si no se proporciona una atención de urgencia o quirúrgica, donde no existe tiempo necesario para realizar PCR como prueba COVID-19, o donde simplemente no se puede demostrar una trazabilidad del paciente, o si bien éste es sintomático o asintomático se recomiendan las precauciones extremas de manejo y monitorear, tomando muestras posteriores al procedimiento quirúrgico y poder determinar los niveles de precaución que también pueden variar con el correr del tiempo.

Dentro de este tipo de fracturas se encuentran las que involucran lesiones a nivel de la base de cráneo, aquellas que comprimen el nervio óptico o aquellas en que hay alto riesgo de compromiso de vía aérea ya sea por el rasgo de fractura o por las condiciones de base del paciente (Zhou et al., 2020).

A su vez, lesiones que no sean urgentes y no determinen secuelas funcionales y/o estéticas, se deben observar y diferir su resolución hasta contar con todos los exámenes necesarios. Algunas lesiones faciales no son de una urgencia absoluta, si pueden requerir una intervención quirúrgica en el corto plazo para evitar secuelas como infecciones, deformidades permanentes y alteraciones estéticas.

Lo que se traduce, en que, dentro del marco de un trauma facial, donde el contacto, debido a la zona afectada es de mayor riesgo, se exigen procedimientos y toma de decisiones más estrictas; siendo sumamente importante el aporte de los distintos protocolos y recomendaciones avaladas por la literatura y en algunos casos por la experiencia de los equipos clínicos y centros hospitalarios.

Una buena medida es la categorización que realiza la Universidad de Stanford y de Minnesota (descrita anteriormente) sobre las medidas a considerar dependiendo del caso, sin poner en duda el uso de los elementos de protección personal; evaluando el uso de purificadores de aire, y categorización del estatus COVID-19 del paciente (Hsieh et al.; Vukkadala et al.).

Dentro de las medidas que se incluyen inicialmente respecto a los elementos de protección perso- nal, son el uso de respiradores, o en su defecto, la utilización de mascarillas N95 certificadas por el Nationallnstitute for Occupational Safety and Health (NIOSH) o símiles estandarizadas frente al uso de aerosoles en los procedimientos (Thomas et al., 2020).

La AO CMF describió estrategias alternativas como el uso de mascarillas FFP3 o FF2 combinadas con antiparras y trajes de protección, incluso en ambientes de consulta por el alto nivel de carga viral en las estructuras analizadas por parte de las especialidades de Anestesiología, Cirugía de Cabeza y Cuello, Cirugía Maxilofacial y Otorrinolaringología (Grant et al., 2020a).

La Academia Americana de Otorrinolaringología en conjunto con la sociedad de cirujanos de cabeza y cuello, en su aporte a la protocolización de casos quirúrgicos, indicaron que lo ideal es que el equipo quirúrgico ingrese al pabellón 20-30 minutos posteriores a la intubación y la generación de aerosoles que ésta pudiese generar, agregando por ejemplo que las traqueotomías sólo se debiesen realizar en pacientes preferiblemente COVID-19 negativos y demostrable.

Los procedimientos deben ser limitados, en el caso de traumatismos faciales, a fracturas en que su aplazamiento o no resolución pueda afectar el resultado final (Grant et al., 2020b; Zeng et al., 2020).

Dentro del marco de las recomendaciones a seguir, la AO CMF desarrolla un artículo "International Task Force Recommendations on Best Practices for Maxillofacial Procedures during COVID-19 Pandemic" enumerando una serie de medidas descritas según región tercio facial de la fractura o procedimiento (Grant et al., 2020a).

Como medidas generales, se recomienda reducir los equipos de trabajo, preferir procedimientos cerrados si no se requiere una fijación interna, disminuir la irrigación y protocolos de generación de aerosol (Fig. 1).

\section{Tercio inferior/ fracturas mandibulares}

- Uso de tornillos autoperforantes en MMF.

- Bisturí monopolar para incisiones en mucosa.

- Cauterizar para hemostasia.

- Usar instrumentos de baja velocidad.

- Preferir fijación intermaxilar si es que fractura requiere tratamiento y luego de transcurrido el tiempo necesario usar abordaje transcutáneo. 
YÉVENES, S. F.; DIAZ, R. M. \& DONODO, H. F. Recomendaciones para la categorización y manejo de pacientes con traumatismos faciales durante pandemia por COVID-19 (SARS-CoV-2). Revisión de la literatura. Int. J. Odontostomat., 15(1):31-35, 2021

Tercio Medio

- Considerar el uso de tornillo de Carroll-Girard, evitando incisión intraoral

- Evitar irrigaciones repetidas

- Tornillos autoperforantes

\section{Tercio Superior/ Seno Frontal \\ -Considerar aplazamiento}

Evitar procedimientos endonasales debido a generación de aerosoles
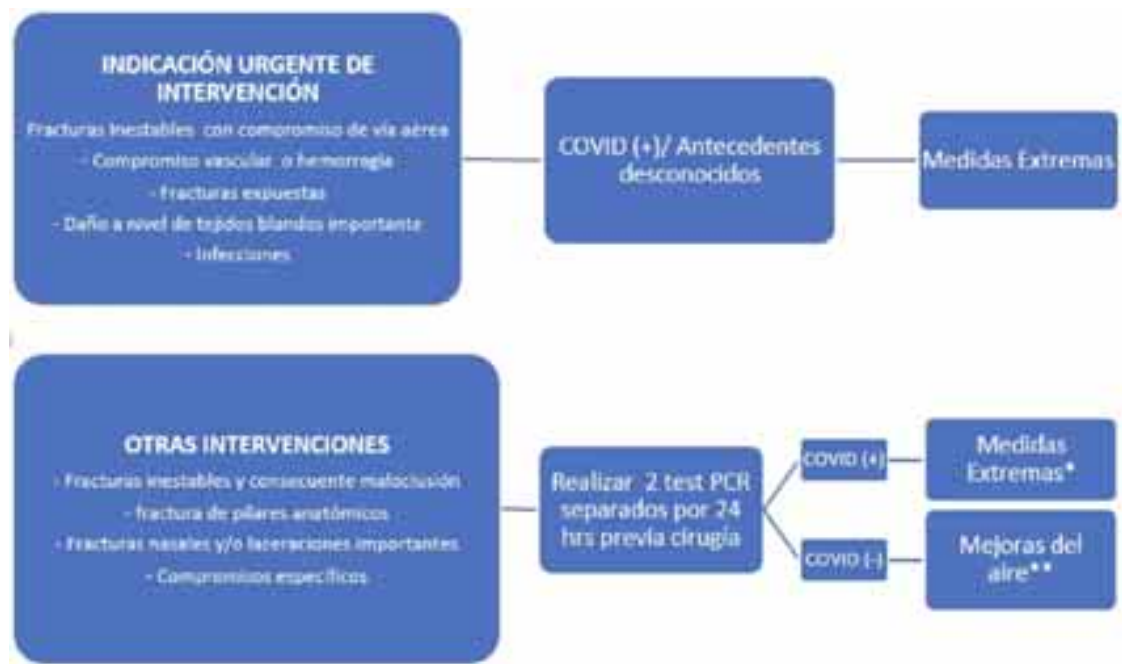

Fig. 1. Sugerencias al momento de la atención en tiempos de COVID-19.

*Uso de motor purificador de aire, ropa clínica resistente a fluidos, guantes quirúrgicos, mascarilla N95 o FFP2, mascarilla quirúrgica, protector facial u ocular, gorro y guante.

**Uso de mascarillas N95 o FFP2, protector facial u ocular, ropa resistente a fluidos, gorro y guantes

\section{CONCLUSIONES}

Dentro del impacto que ha provocado esta pandemia a nivel de los servicios de salud, es que se hace imperioso el cuidado y actualización en los criterios de categorización de pacientes afectados por traumas faciales, debido a lograr un cuidado del paciente y personal de salud y así poder optimizar todos los recursos disponibles.

Respecto al manejo de un trauma facial y las precauciones que hay que tomar, se le agrega en estas situaciones un especial cuidado, debido a las zonas comprometidas que en gran parte pueden comprometer la vía aérea y/o la generación de aerosoles para su resolución.

También hay que tomar en cuenta los equipos multidisciplinarios que muchas veces participan de estos procesos, por lo cual se hace imperioso y de suma importancia la categorización de los pacientes y a su vez respetar los protocolos que se encuentran en la literatura.
YÉVENES, S. F.; DIAZ, R. M. \& DONODO, H. F. Recommendations for the categorization and management of patients with facial trauma during a COVID-19 (SARSCoV-2) pandemic. Literature review. Int. J. Odontostomat., 15(1):31-35, 2021.

ABSTRACT: Facial trauma constitutes itself a problem of relevance in highly complex hospital centers, due to the functional and aesthetic consequences that it may entail. That is why diagnosis is needed, as an assertive response and proper management of it, mostly in times of a pandemic disease like COVID-19. Different protocols were reviewed, regarding the categorization and management of this pathology, taking into account the pandemic situation that affects us. The objective of this article is to present and bring these protocols closer together, and then, be a contribution to the different professionals dedicated to facial trauma treatments at hospital centers, regarding the safety measures with the staff and also its patients

KEY WORDS: COVID-19; SARS-CoV-2, facial trauma, protocol. 
YÉVENES, S. F.; DIAZ, R. M. \& DONODO, H. F. Recomendaciones para la categorización y manejo de pacientes con traumatismos faciales durante pandemia por COVID-19 (SARS-CoV-2). Revisión de la literatura. Int. J. Odontostomat., 15(1):31-35, 2021.

\section{REFERENCIAS BIBLIOGRÁFICAS}

American College of Surgeons. COVID-19 Guidelines for Triage of Emergency General Surgery Patients. Chicago, American College of Surgeons, 2020a. Disponible en: https://www.facs.org/ covid-19/clinical-guidance/elective-case/emergency-surgery

American College of Surgeons. COVID-19: Guidance for Triage of Non-Emergent Surgical Procedures. Chicago, American College of Surgeons, 2020b. Disponible en: https://www.facs.org/covid19/clinical-guidance/triage

Federazione Nazionale degli Ordini dei Medici Chirurghi e degli Odontoiatri. Elenco dei Medici caduti nel corso dell'epidemia di Covid-19. Roma, Federazione Nazionale degli Ordini dei Medici Chirurghi e degli Odontoiatri, 2020. Disponible en: https:// portale.fnomceo.it/elenco-dei-medici-caduti-nel-corsodellepidemia-di-covid-19

Grant, M.; Buchbinder, D.; Dodson, T. B.; Fusetti, S.; Leung, M. Y. Y.; Sánchez Aniceto, G.; Schramm, A.; Strong, E. B. \& Wolvius, E. AO CMF International Task Force Recommendations on Best Practices for Maxillofacial Procedures During COVID-19 Pandemic. Craniomaxillofac. Trauma Reconstr., 13(3):151-6, 2020.

Grant, M.; Schramm, A.; Strong, E. B.; Buchbinder, D.; Ellis, E.; Wolvius, E. \& Sánchez Aniceto, G. AO CMF International Task Force Recommendations on Best Practices for Maxillofacial Procedures during COVID-19 Pandemic. Davos, AO CMF, 2020. Disponible en: https://www.aofoundation.org/-/media/project/ aocmf/aof/documents/ao-cmf-covid19-guidelines.pdf

Hsieh, T. Y.; Dedhia, R. D.; Chiao, W.; Dresner, H.; Barta, R. J.; Lyford-Pike, S.; Hamlar, D.; Stephan, S. J.; Schubert, W. \& Hilger, P. A. A Guide to Facial Trauma Triage and Precautions in the COVID-19 Pandemic. Facial Plast. Surg. Aesthet. Med., 22(3):164-9, 2020.

Kaplan, A.; Georgiev, P.; Enright, M. \&, Oakes, C. What to do if you are concerned you have COVID-19, according to state health departments. NBC News, March 14, 2020. Disponible en: https:/ /www.nbcnews.com/health/health-news/coronavirustestinginformation-covid-19-tests-according-state-healthdepartments$\mathrm{n} 1158041$

Minder, R. \& Peltier, E. Virus Knocks Thousands of Health Workers Out of Action in Europe. The New York Times, March 24, 2020. Disponible en: https://www.nytimes.com/2020/03/24/world/ europe/coronavirus-europe-covid-19.html

Organización Mundial de la Salud (OMS). Coronavirus. Geneva, Organización Mundial de la Salud, 2020. Disponible en: https:// www.who.int/health-topics/coronavirus\#tab=tab_1

Rubio-Perez, I.; Badía, J. M.; Mora-Rillo, M.; Quirós, A. M.; García Rodríguez, J.; Balibrea, J. \& Grupo Cirugía-AEC-COVID-19ه COVID-19: Conceptos clave para el cirujano. Cir. Esp., 98(6):3109, 2020.

Sociedad Chilena de Cirugía Pediátrica. Recomendaciones para manejo paciente quirúrgico con COVID-19 +. Santiago de Chile, Sociedad Chilena de Cirugía Pediátrica, 2020. Disponible en: https://www.schcp.cl/wp-content/uploads/2020/04/ RECOMENDACIONESPARAMANEJOPACIENTEQUIRU\% CC\%81RGICOCONCOVID-19.pdf

Thomas, J. P.; Srinivasan, A.; Wickramarachchi, C. S.; Dhesi, P. K.; Hung, Y. M. A. \& Kamath, A. V. Evaluating the national PPE guidance for NHS healthcare workers during the COVID-19 pandemic. Clin. Med. (Lond.), 20(3):242-7, 2020.

Vukkadala, N.; Qian, Z. J.; Holsinger, F. C.; Patel, Z. M. \& Rosenthal, E. COVID-19 and the otolaryngologist: preliminary evidencebased review. Laryngoscope, 2020. DOI: https://www.doi.org/ 10.1002/lary.28672
Zeng, L.; Su, T. \& Huang, L. Strategic plan for management in oral and maxillofacial surgery during COVID-19 epidemic. Oral Oncol., 105:104715, 2020

Zhou, F.; Yu, T.; Du, R.; Fan, G.; Liu, Y.; Liu, Z.; Xiang, J.; Wang, Y.;Song, B.; Gu, X.; et al. Clinical course and risk factors for mortality of adult inpatients with COVID-19 in Wuhan, China: a retrospective cohort study. Lancet, 395(10229):1054-62, 2020.

Dirección para correspondencia:

Francisco Yévenes Souper

Servicio de Cirugía Oral y Maxilofacial

Hospital San Juan de Dios

Avenida Portales 3239

Santiago

CHILE

Email: fyevenessouper@gmail.com 